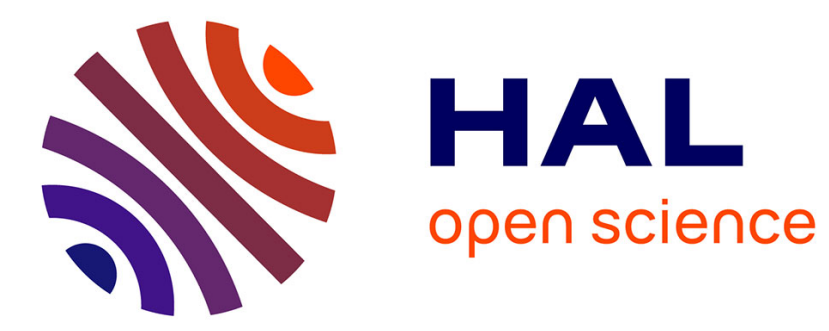

\title{
Un diagnostic pour re-connaître et territorialiser? Contribution à la construction d'un projet de Parc naturel régional dans les Baronnies (Drôme, Hautes-Alpes)
}

\author{
Emmanuel Roux, Romain Lajarge, Mathieu Esterni
}

\section{To cite this version:}

Emmanuel Roux, Romain Lajarge, Mathieu Esterni. Un diagnostic pour re-connaître et territorialiser? Contribution à la construction d'un projet de Parc naturel régional dans les Baronnies (Drôme, HautesAlpes). Géocarrefour - Revue de géographie de Lyon, 2005, 80 (2), pp.133-143. halshs-00329209

\section{HAL Id: halshs-00329209 \\ https://shs.hal.science/halshs-00329209}

Submitted on 16 Oct 2008

HAL is a multi-disciplinary open access archive for the deposit and dissemination of scientific research documents, whether they are published or not. The documents may come from teaching and research institutions in France or abroad, or from public or private research centers.
L'archive ouverte pluridisciplinaire HAL, est destinée au dépôt et à la diffusion de documents scientifiques de niveau recherche, publiés ou non, émanant des établissements d'enseignement et de recherche français ou étrangers, des laboratoires publics ou privés. 


\section{Un diagnostic pour re-connaître et territorialiser ? Contribution à la construction d'un projet de Parc Naturel Régional dans les Baronnies (Drôme, Hautes-Alpes).}

Emmanuel Roux, UMR Pacte, Territoires, Romain Lajarge, UMR Pacte, Territoires Mathieu Esterni, I-tem consultant

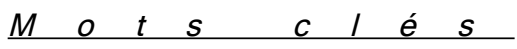

Diagnostic territorial, intentionnalité, démarche méthodologique, dispositif cognitif, représentations territoriales, acteurs, discours, périmètre, actions, territorialisation, Baronnies.

$$
\underline{R} \quad \dot{e} \quad s \quad u \quad m \quad e ́
$$

A l'heure où le projet de territoire est devenu un objet assez consensuel au succès affirmé, il nous semble nécessaire de rendre compte d'expériences vécues et de quelques réflexions que l'on peut tirer de la manière dont de tels projets sont construits. La présente contribution s'appuie sur l'expérience d'un travail d'étude conduit pendant près d'un an sur le territoire des Baronnies dans le cadre de la mise en œuvre d'un projet de Parc Naturel Régional.

Cette contribution propose en préambule, de discuter le positionnement des acteurs en présence, ainsi que les intentions (explicites ou implicites) associées, dans le cas présent, à la réalisation d'un diagnostic de territoire (entendu ici comme dispositif contextualisé de porté à connaissance des ressources territoriales en vue de la construction d'un projet de territoire). II s'agit ici de discuter les dispositifs méthodologiques successivement mis en œuvre. Dans une première phase, ont été recherchés les arguments qui permettaient de donner du sens au projet de ce territoire ; tant dans l'appréhension des représentations des acteurs locaux dans les organisations chargées du développement, que dans les représentations et le vécu quotidien des habitants des Baronnies. Pour se faire, ce travail a été réalisé grâce à des questionnaires, des entretiens, la réalisation de représentations cartographiques et l'analyse des documents programmatiques existants (phase A). Une seconde phase (phase B) a consisté à élaborer un diagnostic décliné thématiquement et présentant les atouts et faiblesses du territoire, les opportunités et menaces pour celui-ci. La phase $\mathrm{C}$ a proposé de partir de la prise en considération de configurations et stratégies territoriales pour définir un périmètre de candidature. Elle présente enfin un dispositif définissant des actions démonstratives susceptibles d'être mise en œuvre sur le territoire.

Le texte proposé ici constitue à la fois un témoignage du travail conduit dans les Baronnies et une proposition d'analyse de ce dispositif méthodologique susceptible de contribuer à la réflexion sur les modalités discursives de la construction territoriale et leurs effets sur le système d'acteurs territorialisés. Un double processus permettant l'éclosion d'un projet de territoire à partir d'un diagnostic territorial est proposé : instituer et constituer le territoire par le projet. 
II n'existe pas de diagnostic objectif et sans objectifs. Décrire un territoire relève d'une sélection, d'une saisie d'objets, et implique donc de se dessaisir d'autres objets. La sélection et la hiérarchisation de ces objets sont inhérentes au processus de diagnostic territorial. Comme nous y invite André Corboz, pour tenter d'éviter cet " espèce d'inventaire maniaque (néopaléopositiviste) " (Corboz, 2001) et considérer le territoire comme palimpseste, diagnostiquer revient à faire deux descriptions. La première, instrumentale, sert à fonder le projet ; la seconde, rhétorique, sert à le communiquer, " sorte de preuve anticipée de sa pertinence ». Si l'on s'inscrit dans une perspective constructiviste, si l'on souhaite démontrer que même la phase décrite par les acteurs territoriaux comme objectivante est d'abord une phase de construction politique du réel, si l'on envisage la description comme une opération pour rendre visible les intentions préalables, alors la posture d'observation participante s'impose rapidement.

Comment ce processus se déroule-t-il ? Qui, quand, comment les acteurs interagissent pour produire, dans le temps du diagnostic, des images et des figures, des orientations et des préconisations, des propositions d'actions et des programmations ? Où ce processus s'inscrit-il dans l'espace de la négociation comme dans l'espace euclidien visé par le projet? Sur quels périmètres vont-ils s'entendre et pourquoi celui-là ?

L'étape du diagnostic dans la construction d'un projet de territoire présente de nombreux avantages pour comprendre à la fois l'intention, le projet et l'action : il se déroule en général sur un délai déterminé, il fait l'objet d'une commande, il relève de l'exceptionnalité et il offre des occasions nouvelles pour reformuler l'existant. Pour ces raisons, il est proposé d'observer de l'intérieur un processus de diagnostic territorial et pour que la démonstration soit étayée par des observations les plus proches possibles " de ce qui s'est passé », nous nous appuyons sur la réalisation de " l'étude de faisabilité et d'opportunité pour la création d'un Parc Naturel Régional dans les Baronnies » pour laquelle la région Rhône-Alpes avait lancé un appel à projet. Le déroulement de ce travail et l'analyse que nous en faisons font l'objet du texte ci-après.

\section{Une étude pour un projet de territoire dans les Baronnies}

A partir de 1995, des acteurs socio-économiques des Baronnies (partie drômoise) engagent une réflexion sur le devenir du territoire des Baronnies, couvrant près de 250000 hectares des Préalpes méditerranéennes, situé aux confins des Régions Rhône-Alpes et Provence-Alpes-Côte d'Azur. Le constat d'une situation de déprise rurale marquée, la nécessité de maintenir les activités économiques, une agriculture de qualité et un cadre de vie agréable les amène à évoquer l'idée d'un parc naturel régional. En 1998, le Groupement pour la Promotion et l'Expansion du Nyonsais et des Baronnies (GPENB), composés de socioprofessionnels du sud de la Drôme, se saisit de ce dossier et développe des contacts avec la Région Rhône-Alpes. Dès lors, cette initiative s'élargit en associant des acteurs locaux des Hautes-Alpes, des parlementaires et des élus locaux. En 2001, l'Association d'Etude pour la création d'un Parc naturel régional des Baronnies voit le jour. Face à cette dynamique locale, les Conseils Régionaux de Rhône-Alpes et de Provence-Alpes-Côte d'Azur décident en 2002 d'engager une étude d'opportunité et de faisabilité d'un Parc naturel interrégional dans les Baronnies et lancent en 2003 une consultation pour la réalisation de ce travail ${ }^{1}$. Organisée en trois phases complémentaires respectivement dénommées " opportunité » (phase A), "faisabilité » (phase B) et " définition d'un périmètre d'étude et d'actions démonstratives " (phase $\mathrm{C}$ ), les résultats de cette étude devaient permettre aux deux Régions de se prononcer sur la pertinence de l'outil « parc naturel régional » pour ce territoire et fixer, le cas échéant, les modalités d'engagement de la procédure officielle, notamment le périmètre d'étude.

Ce texte propose ici de discuter, dans un premier temps, les contextes de réalisation de ce diagnostic de territoire. Dans un second temps, il s'agit d'expliciter la mise en œuvre de la démarche méthodologique appliquée ${ }^{2}$. Dans un troisième temps, il est proposé de repérer ce qui, dans cette expérience de diagnostic de territoire, permet l'élaboration d'un cadre privilégié pour l'action et donc l'émergence d'une intentionnalité collective permettant de mettre en œuvre un projet. Enfin, pour conclure, il s'agit de montrer comment ce processus particulier, de diagnostic, permet une re-

\footnotetext{
${ }^{1}$ Le Centre d'Etudes et de Recherches sur les Montagnes Sèches et Méditerranéennes (CERMOSEM - Territoires, UMR PACTE) a fait le choix dans ce contexte, de répondre à cette consultation en impliquant une équipe pluridisciplinaire, majoritairement composée de géographes et des étudiants en formation de l'Institut de Géographie Alpine de Grenoble.

2 Initialement prévue sur une durée de sept mois de septembre 2003 à mars 2004, le travail s'est en réalité déroulé pendant près d'une année entre septembre 2003 et juillet 2004. En Décembre 2004, était organisée sur le territoire des Baronnies, une présentation publique et synthétique des résultats de ce travail.
} 
connaissance et par elle une territorialisation nouvelle ; cette reterritorialisation trouvant dans le projet l'occasion de s'instituer et de se constituer.

\section{Diagnostic et intentions croisées}

La proposition d'une démarche méthodologique susceptible de contribuer à la construction d'un projet de territoire n'est pas neutre en soi parce qu'il n'existe pas de projet de territoire sans intentions (Lajarge R., 2000). L'élaboration de ce que l'on nommera de façon générique "diagnostic de territoire ", ne peut-être dissociée des intentions sous-jacentes à la réalisation d'un tel projet. Cette démarche s'inscrit dans des contextes contraignants : celui de la procédure Parc Naturel Régional, celui d'une commande effectuée par des Régions, celui d'une forte demande de participation d'acteurs de territoire et celui d'incertitudes autour d'un tel projet. Aussi faut-il s'adapter à ces contextes dans la mesure où « il n'existe bien sûr pas de méthode unique » (Guéringer A., Perret J., Baud G., 1993) ${ }^{1}$. Une telle démarche oblige à un travail auto-réflexif continuel et invite à interpréter la pratique de recherche dans la mesure où les procédés de validation habituellement utilisés dans un travail scientifique sont venus se heurter plus d'une fois aux procédés de validation imposés par le commanditaire. Se pose alors pour le chercheur la signification de ces distorsions et l'analyse a posteriori de cette expérience.

\subsection{Une double proximité avec l'opérateur du diagnostic et avec les acteurs régionaux commanditaires}

La première volonté pour comprendre ce qu'est le territoire étudié, ses enjeux, sa possible inscription dans une procédure de type Parc Naturel Régional a été de s'approcher au plus près "des protagonistes ». Dans le cas présent, cette proximité est double.

Elle tient d'une part à une implication du chercheur dans la procédure en tant qu'acteur " producteur » de l'étude. Compte tenu notamment des antécédents en matière de travaux de recherches ${ }^{2}$ sur le territoire des Baronnies, les deux Régions ont choisi de retenir un prestataire universitaire pour porter un regard neuf sur "les ressources objectives" des Baronnies. Le chercheur, en acceptant d'endosser ce rôle, répond à une commande plus ou moins normée et se donne la possibilité d'être en situation privilégiée pour décrypter les logiques d'acteurs et la mise à jour de ce qu'est le sens de ce territoire pour chacun des protagonistes.

La seconde proximité tient dans le souci méthodologique affirmé dans la réponse à l'appel d'offre. Le protocole méthodologique a été fixé en amont par une équipe restreinte. Pour chacune des phases de travail, il a été débattu lors de réunions techniques (regroupant les commanditaires du travail et les représentants du territoire). Cette proposition d'aller-retour a été envisagée comme une coconstruction avec le Comité de pilotage ${ }^{3}$. Ce travail de composition a impliqué parfois quelques reformulations et ré-argumentations qui n'étaient pas toujours que formelles.

Méthodologiquement, le choix avait été fait de donner une grande place à l'écoute des acteurs via la réalisation d'une enquête auprès de la population, la conduite d'entretiens auprès d'acteurs politiques, associatifs et socio-économiques, l'organisation de rencontres-débats sur le terrain et la présence assidue aux réunions publiques. En stimulant l'énonciation par les acteurs de ce qu'est le territoire des Baronnies, la démarche privilégie l'expression puis l'interprétation de ce que les acteurs impliqués ou concernés par la procédure disent, pensent, se représentent du territoire.

\footnotetext{
${ }^{1}$ Cf. Chapitre II « Une démarche et des méthodes à adapter » in Guéringer A., Perret, J., Baud G., 1993, Diagnostics à l'échelle de petites régions rurales : démarches, méthodes et sources d'informations », Coll, Etudes du CEMAGREF, Série Production et Economie agricoles, $\mathrm{n}^{\circ} 5$, pp. 25-28.

${ }^{2}$ Le CERMOSEM est installé depuis 1994 en Ardèche en tant qu'antenne de l'Institut de Géographie Alpine, Université Joseph Fourier et du laboratoire Territoires (UMR Pacte). Ces membres participent à des programmes de recherche-développement, travaux d'expertises sur la thématique du développement territorial. Ainsi, de nombreux chercheurs sont venus enquêter, réfléchir, analyser ce qui se passaient dans ces territoires, de nombreux étudiants ont sillonnés les routes de l'Ardèche et de la Drôme, de nombreux stages ont été organisés sur ces terrains. Ces antécédents, la connaissance du territoire et de certains acteurs constituent vraisemblablement des raisons pour lesquelles les Régions Rhône-Alpes et PACA ont opté pour que ce travail soit conduit par le CERMOSEM : ils connaissaient la présence universitaire engagée dans le sud de Rhône-Alpes et son rôle en matière d'animation des débats et des réflexions sur le développement territorial.

${ }^{3}$ Le Comité de Pilotage était composé d'élus et de techniciens régionaux, de représentants des services de l'Etat et de représentants du territoire.
} 


\subsection{Quels problèmes pose le principe de « rester en surface pour décrire les enjeux de fond $॥$ ?}

Le travail de diagnostic de territoire est une étape (parmi d'autres) de recomposition du système d'acteurs. En rappelant cette évidence de la recherche impliquée, nous ne sommes pas dupes de ce que le travail avec les acteurs permet de révéler. En tant qu'acteur principal de la procédure de projet de PNR les Baronnies pendant un semestre ${ }^{1}$, l'équipe de chercheurs se devait a priori de rester plutôt à la " surface des choses » car la dynamique politique autour d'un tel projet réclamait une grande prudence. Et pourtant, la phase de diagnostic, aboutissant à un avis sur la faisabilité ou non d'un tel projet, ne pouvait pas faire l'économie d'un travail sur les enjeux de fond.

La " contrainte " de devoir évoluer a priori " en surface " tient aux cadres " pré-établis ", formels et informels : au respect d'une procédure officielle (décret de 1994) où les Régions sont considérées comme " chef de file » et au respect d'une commande définie (l'étude d'opportunité et de faisabilité) qui doit aussi composer avec les acteurs du territoire. Concernant la procédure de création d'un Parc naturel régional, les Régions, porteuses du projet ${ }^{2}$, sont invitées à s'assurer de la pertinence d'un projet de création en choisissant un périmètre d'étude, en le justifiant du triple point de vue de la richesse patrimoniale, de la fragilité environnementale et des perspectives envisageables pour y conduire un projet permettant un développement durable du territoire. Dans le même temps, les acteurs du territoire continuent à se mobiliser autour de ce projet. Les Régions doivent alors porter une attention particulière aux élus locaux et veiller à coller au mieux aux attentes des groupes constitués et au respect des équilibres politiques locaux. Dans cette phase amont sont susceptibles de se jouer au moins trois enjeux de fond (Lajarge R., Roux E., 2004) :

- celui, politique, qui amène les Conseils municipaux à voter favorablement ou défavorablement la charte une fois la procédure achevée ;

- celui, technique, qui consiste à rédiger un texte de cadrage à valeur contractuelle (la Charte), qui par le jeu de la sélection des déclarations initiales d'intentions peut faire ou défaire les groupes d'acteurs participants à la démarche ;

- celui enfin, stratégique, qui tient dans la définition de la structure porteuse de la démarche, de son poids (en terme de budget de fonctionnement c'est-à-dire de chargés de mission) et de son statut (presque obligatoirement un syndicat mixte), pouvant accélérer ou ralentir le démarrage de la procédure.

- Dans l'exercice du diagnostic, le prestataire est invité, implicitement au moins, à ne pas modifier l'ordre établi des choses et donc à rester plutôt « à la surface ». Le diagnostic est en quelque sorte "contraint» par l'impérieux souci de maintenir les équilibres et de ne pas risquer d'hypothéquer, dès le départ, le projet.

La seule perspective de la démarche de diagnostic, avant même sa mise en œuvre, est donc déjà un exercice saturé d'intentions multiples, parfois contradictoires. L'idée même de diagnostic introduit, bien avant de révéler son contenu, des interactions subtiles, en amont de la réalisation, entre ceux qui sont à l'origine même de la définition de la commande, ceux dont on est obligé de prendre en compte la présence et ceux qui portent des enjeux contextuels. Une fois ces positions de départ en partie révélées, la démarche peut se dérouler. Dans notre cas de figure, le diagnostic devra comporter différentes étapes, mais devra surtout se penser en lien étroit avec les acteurs locaux pour être au plus près du territoire.

\section{Un diagnostic participatif pour re-connaître ? Ou comment « faire dire ce qu'est le territoire $»$.}

A l'instar de quelques travaux décrivant et analysant des méthodes éprouvées de diagnostic (Conseil Général du Génie Rural, des Eaux et des Forêts, 1993 ; Rigaldies B., 1996 ; Mairie-Conseil, 1997 ; Comité de Liaison des Comités de Bassins d'Emploi, DATAR, 1997 ; Cuaresma M., Pecqueur B., 1997), nous reconnaissons pleinement la pertinence de l'adjectif " participatif ». Mais, nous ne l'aborderons ni au titre de l'injonction réglementaire qui introduit dans les derniers dispositifs législatifs

\footnotetext{
${ }^{1}$ Durée de la première phase de diagnostic qui a privilégié la rencontre avec les acteurs du territoire.

2 Dans le cadre de la procédure de Parc Naturel Régional, le Ministère de l'Ecologie et du Développement Durable considère les Régions comme porteuses du projet.
} 
plus de " concertation », d' " enquête publique », d'« ouverture à la société civile », ... ni au titre de son omniprésence dans le champ interprétatif (même sous ses dérivés "gouvernance " ou "coconstruction »), mais en tant qu'argument de la commande des Conseils Régionaux. Le diagnostic, ici, devait appréhender les motivations, les intérêts des acteurs et les enjeux du territoire. Pour se faire, une enquête sur ce que les gens pensaient de ce projet, ce qu'ils disaient à propos de la perspective d'avoir un Parc dans les Baronnies, ce que l'on pouvait recenser sur cette idée était considérée comme une dimension participative du diagnostic.

\subsection{Un pré-diagnostic pour rendre compte des conditions propices à l'émergence d'un projet de territoire}

Cette étape de diagnostic ${ }^{1}$ a proposé d'appréhender les motivations et les intérêts des acteurs selon deux registres complémentaires : les regards et opinions des acteurs et les dynamiques territoriales existantes (les structures institutionnelles, leurs héritages, leurs expériences et leurs souhaits).

\subsubsection{Les mots des acteurs pour dire ce qu'est le territoire}

Pour répondre à l'objectif (inscrit dans la " commande ») de " placer au centre de cette démarche les acteurs du territoire et donner une place privilégiée à leur écoute », trois dispositifs d'investigation sont mis en place de façon concomitante : un questionnaire, des entretiens et une présence continue tout au long de la démarche.

Considérant la population comme actrice potentielle du développement du territoire (Gumuchian et al., 2003), la réalisation d'une enquête par questionnaires (au nombre de 240) a permis de qualifier ce qu'était, au regard de la population, la spécificité des Baronnies (Lajarge R., Roux E., 2004)2. Une seconde partie du questionnaire était davantage consacrée à "la connaissance de ce qu'était un PNR » ; un support cartographique était alors adjoint au questionnaire et chacun était invité à dessiner ou tracer « son périmètre idéal ».

Le cahier des charges stipulait explicitement qu'il conviendrait de procéder à des entretiens auprès d'acteurs du territoire ciblés. Rappelons là le souci des commanditaires d'observer une attention particulière à l'égard des «figures » locales. Le choix (validé par le comité de pilotage) a donc été fait de procéder à des entretiens semi-directifs auprès d'un panel d'acteurs (une vingtaine) du territoire ${ }^{3}$. II s'agissait ainsi de veiller à avoir une représentativité géographique (drômoise et haute-alpine) et une représentativité d'opinions (politiques, institutionnelles, socioprofessionnelles, culturelles...). La grille d'entretien proposait à chaque acteur de se prononcer sur « le territoire des Baronnies ; son évolution en terme d'activités, en terme de développement, en terme d'organisations ; ses enjeux; sur l'idée d'un projet de PNR ou encore les conditions de sa faisabilité,... ».

Un troisième dispositif d'investigation s'est progressivement mis en place au cours de cette étape du travail. II a consisté à demeurer à l'écoute en continu des acteurs du territoire et ce en dehors des deux dispositifs précédents; soit en étant présent sur le territoire lors de réunions publiques (organisées par des associations de développement), soit en se rendant disponible auprès d'acteurs individuels et collectifs souhaitant s'exprimer par courrier, entretiens téléphoniques, rencontres ou entretiens considérés comme plus informels. Cette présence a permis, en outre, d'entendre des acteurs souhaitant exprimer, en marge des cadres conventionnels de l'étude, leurs opinions, leurs espoirs mais également leurs craintes voire leurs revendications.

Ces trois dispositifs complémentaires ont permis de lancer la démarche de diagnostic, de constituer un socle premier « de représentations et de regards croisés sur le sens du territoire des Baronnies et de l'idée de projet de Parc Naturel Régional sur ce territoire ».

\footnotetext{
${ }^{1}$ Nous nous référons ici au document de valorisation de cette étude « La construction d'un projet de territoire. Un projet de Parc Naturel Régional pour le territoire des Baronnies », ss dir. E. Roux, H Gumuchian, 2005, UMR Pacte, Territoires-CERMOSEM.

2 Cf. pp. 167 à 169 in Lajarge (R.), Roux (E.), 2004, « L'activation des ressources par le projet : les Baronnies en recherche de nouvelles légitimités », in "La notion de ressource territoriale », Montagnes Méditerranéennes, n²0, pp. 165-176.

${ }^{3}$ Le panel d'acteurs du territoire était composé de parlementaires, élus locaux, représentants d'associations (de développement à l'initiative du projet de Parc Naturel Régional, de protection de la nature, de fédération (de chasse notamment), d'acteurs socioprofessionnels.
} 


\subsubsection{S'appuyer sur l'expérience acquise par les collectivités territoriales}

Le souhait de vouloir vérifier les motivations des acteurs quant à la mise en place d'un tel projet de territoire ne pouvait se satisfaire exclusivement du discours des acteurs. Aussi, il nous est apparu indispensable de compléter le premier volet du diagnostic par une démarche visant aussi à appréhender les intentions et intérêts au travers des organisations, des actions entreprises sur le territoire depuis plusieurs années en matière de développement. Ce second volet a donc été celui de l'analyse des dynamiques territoriales et des expériences antérieures sur laquelle ce territoire s'appuie. II consistait en l'analyse de documents programmatiques, de bilans d'activités, de données relatives aux actions de développement collectés auprès des collectivités territoriales et/ou des structures de développement, complété par la réalisation de quelques entretiens semi directifs. II propose une relecture des programmes de développement, des coopérations passées, des structurations et organisations, des actions conduites sur le territoire. Cette investigation constituait ainsi une contribution à l'évaluation de "l'engagement » passé et présent du territoire dans l'action et finalement à sa capacité à « ressembler » dans le futur à ce que sont les Parcs.

\subsubsection{L'aire du diagnostic : comment rendre acceptable un futur périmètre}

Après avoir appréhendé les motivations des acteurs, l'étape suivante étant consacrée à la mise en évidence des ressources du territoire, il devenait nécessaire de préciser l'aire sur laquelle le diagnostic (thématique) devait se porter. Trois propositions ont été formulées, correspondant à trois pistes possibles. Celles-ci ont été définies à partir de ce que les personnes questionnées avaient ellesmêmes dessinées comme délimitation idéale, sur un fond de carte proposé par les enquêteurs. Les acteurs en présence lors du Comité de pilotage étaient donc invités à faire le choix « d'élargir beaucoup ", " d'élargir un peu » ou " de restreindre " l'aire initialement proposée par les Conseils Régionaux pour faire le diagnostic. En faisant ce premier arbitrage, les acteurs locaux laissaient entrevoir ce que pourraient être leur avis sur le périmètre final du projet de Parc.

Les commanditaires et acteurs du territoire ont étudié avec attention les trois pistes proposées. Mais comme initialement envisagée, l'aire retenue fut bien un mixte entre les différents arguments proposés.

En procédant ainsi, il a été possible de constater la « bonne acceptabilité » de l'idée de Parc Naturel Régional sur ce territoire et de définir une première approximation de ce que pourrait être son périmètre futur. Ce pré-diagnostic met aussi en évidence les incertitudes, les préoccupations quant à la situation et l'évolution de ce territoire, quant au souhait de chacun d'être davantage informé et associé à la construction d'un tel projet. En outre, cette démarche méthodologique a permis l'évocation explicite de spécificités, d'arguments stratégiques et déjà d'enjeux apparemment incontournables : la maîtrise du foncier, le maintien et le développement d'une agriculture de qualité, un certain type de tourisme à développer et/ou à maîtriser, la " perte potentielle de pouvoirs locaux », le maintien des équilibres sur ce territoire,.... Un autre enjeu s'est également imposé : celui de l'organisation, du réagencement des éléments identitaires de ce territoire et des problématiques exprimées in situ. Enfin ce procédé d'auto-suggestion quant à la définition d'une aire idéale pour réaliser le diagnostic a probablement participé à cette re-connaissance initiale du territoire nécessaire à la poursuite du diagnostic.

\subsection{Le diagnostic pour identifier les enjeux du territoire}

Pour rendre compte de la "faisabilité » d'un tel projet (phase B), il fallait mettre en évidence l'existence de ressources territoriales, de pratiques et d'activités (organisation et fonctionnement du territoire), de processus à l'œuvre susceptibles de permettre (voire de justifier ou légitimer) l'inscription du territoire des Baronnies dans une procédure de type PNR. La démarche proposée se déroule alors en trois temps complémentaires (figure 1) : la collecte ou co-construction de données ; l'analyse des " caractéristiques » et dynamiques territoriales selon huit chapitres et la formalisation de synthèses ${ }^{1}$.

\footnotetext{
${ }^{1}$ Ces synthèses devaient être présentées, comme demandées par le commanditaire, sous formes de tableaux « atouts contraintes » / « opportunités - menaces ».
} 


\section{Figure 1: Des données aux enjeux du territoire}

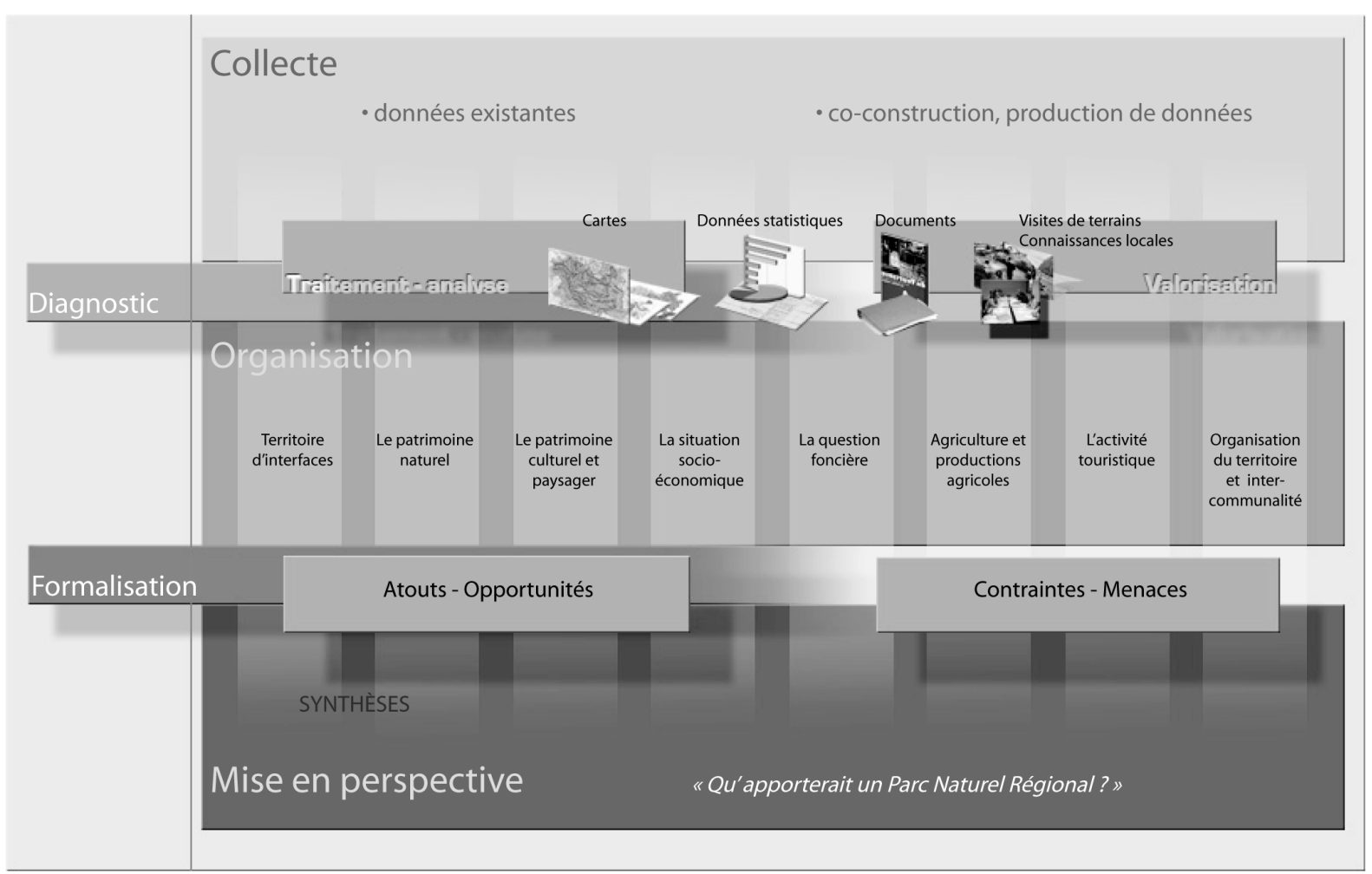

C) E. ROUX, M. ESTERNI - l-tem Consultant, in Roux (E.), Gumuchian (H.), (dir.), 2005, p.87

\subsubsection{Valoriser l'information existante}

L'analyse des ressources et des dynamiques territoriales passe inéluctablement par un inventaire et une collecte de données relatives au territoire considéré. Le cahier des charges, la procédure ainsi que les résultats de la première phase de diagnostic invitaient à quelques orientations thématiques sur lesquelles trois modalités complémentaires de collecte ont alors été mises en place.

La première correspond à la mobilisation de données ${ }^{1}$ issues d'inventaires nationaux ou régionaux et/ou collectées auprès d'organismes ad hoc, de services déconcentrés de l'Etat ou des collectivités territoriales. Pour les commanditaires, ces données sont considérées comme "officielles » et renforcent ainsi le caractère impartial et « scientifique » de la démarche de diagnostic.

La seconde modalité s'appuie sur la collecte de travaux, de rapports effectués sur le territoire ${ }^{2}$. II s'agissait à la fois de mobiliser les connaissances existantes sur et pour le territoire et d'associer l'ensemble de ces structures à la production du diagnostic.

La troisième modalité de collecte de données a consisté à " co-construire " avec les acteurs du territoire l'information. Cette démarche a consisté à mobiliser et à valoriser les savoirs profanes existants en demeurant le plus proche possible des acteurs du territoire dans les domaines considérés; qu'ils soient membres d'associations, personnes ressources identifiées, érudits locaux ou professionnels. Ces collaborations se sont traduites par des rencontres individuelles, des sessions collectives de travail conduite sur le terrain, la réalisation commune d'inventaires (inventaire du

\footnotetext{
$\overline{1}$ Données du recensement général de la population, recensement général de l'agriculture, fichier SIRENE... (INSEE) ; données environnementales (DIREN, Conservatoire national alpin) et associations de protection de l'environnement), etc. - Cf. liste des structures et organismes in « La construction d'un projet de territoire. Un projet de Parc Naturel Régional pour le territoire des Baronnies », ss dir. E. Roux, H Gumuchian, 2005, UMR Pacte, Territoires-CERMOSEM.

2 Elle s'est opérée respectivement auprès d'un syndicat d'aménagement, des communautés de communes, d'organismes de recherches (conservatoire national alpin par exemple), des services déconcentrés de l'Etat, des structures de développement, des chambres consulaires, des associations, etc.
} 
patrimoine $^{3}$; inventaire faunistique et floristique par exemple) et le recueil d'informations via la participation assidue à des réunions-débats thématiques organisées sur le territoire.

\subsubsection{Décliner, pour chaque enjeu, les « atouts et contraintes», les « opportunités et menaces $»$}

L'analyse des données collectées ne devait pas se limiter à « livrer » un inventaire, aussi fourni soit-il, des ressources sur le territoire. Pour cela, trois principes ont été retenus.

Premièrement, il est proposé de décliner les ressources et les dynamiques territoriales selon des entrées thématiques. Cette proposition paraît somme toute assez classique ; mais elle avait pour double intérêt de concilier facilité de lecture et respect des préoccupations exprimées par les acteurs du territoire (et les commanditaires). II s'agissait alors de répondre au vœu d'exhaustivité demandé par le commanditaire dans le cahier des charges et d'opérer une synthèse qui puisse rendre compte de la spécificité de ce territoire. Huit thématiques ont ainsi été fixées: les Baronnies comme territoire d'interface ; le patrimoine naturel ; le patrimoine culturel et paysager ; la situation socioéconomique ; la question du foncier ; l'agriculture et les productions agricoles ; l'activité touristique ; l'organisation du territoire et l'intercommunalité. Chacune de ces thématiques a fait l'objet d'une analyse descriptive des composants du territoire et de ses ressources présentés sous forme "d'atlas " intégrant des représentations cartographiques, graphiques, illustrations iconographiques et courts commentaires.

Mais un atlas ne peut suffire à indiquer en quoi une ressource est susceptible d'être vecteur de développement durable et/ou en "conformité » avec les pré-requis d'un PNR. Aussi, en second lieu, une grille de lecture analytique a été élaborée. Celle-ci proposait de décliner, sous forme de tableaux synthétiques pour chacune des thématiques abordées, ce qui pouvait être considéré comme un " atout » ou une " contrainte » d'une part, " une opportunité » ou " une menace » d'autre part. Cette grille classique a permis la mise en exergue de ce qui était d'ores et déjà en " adéquation » avec la procédure Parc Naturel Régional ou ce qui était susceptible d'être amélioré par le projet de PNR.

En toute logique, la mise en évidence des ressources du territoire, devait s'accompagner d'un regard prospectif (aussi modeste soit-il) indiquant « ce que pourrait apporter au territoire » un Parc Naturel Régional.

Cette phase de diagnostic dans laquelle se jouent à la fois le recueil des représentations et avis des acteurs locaux, la pré-délimitation du futur périmètre et l'inventaire le plus exhaustif possible des enjeux thématiques (avec atlas et grille atouts/contraintes, forces/faiblesses) s'est déroulée dans un pas de temps assez réduit (six mois) avec des moyens traditionnels pour une équipe universitaire (des intervenants spécialisés, des étudiants, des chercheurs) et des méthodes habituelles (recueil de données, vérification, traitements, validation, valorisation). Cependant du point de vue du commanditaire et des acteurs locaux, le fait que le prestataire de service fût universitaire présentait deux spécificités :

- la disponibilité sur le terrain permettant un recueil large des avis et points de vue locaux ; augmentant probablement l'effet « participatif $»^{1}$;

- et la « liberté de propos » des universitaires permettant de ne pas être seulement le porteur de la parole entendue et permettant d'augmenter l'effet « co-constructif » des décisions prises au fur et à mesure de l'avancée du travail de diagnostic.

Bien évidemment, nous ne sommes pas dupes, à ce moment-là, de la dimension double de l'argument " d'innovation " par lequel les acteurs locaux ont accepté un tel diagnostic (à vocation participative, réalisé par des universitaires). Cela permet à la fois de montrer que le territoire innove encore dans sa méthode de travail (comme il l'a souvent fait depuis 20 ans) et que le projet de PNR sera aussi innovant puisqu'il fait l'objet d'une « recherche ». En fait, lorsque arrive le moment de réaliser la partie de diagnostic qui va avoir une implication directe sur les décisions futures, à savoir la délimitation finale du périmètre d'étude du PNR, les enjeux politiques et stratégiques vont prendre toute leur place et les artefacts perdre de leur puissance symbolique.

\footnotetext{
${ }^{3}$ La réalisation d'un inventaire du patrimoine territorial a été réalisée en collaboration avec les acteurs du territoire selon une démarche méthodologique proposée par Pierre-Antoine Landel (CERMOSEM, Territoires, UMR Pacte) dans le cadre d'une session de formation (stage terrain d'une semaine) mobilisant l'ensemble d'une promotion de Licence Professionnelle «Promoteur du Patrimoine Territorial », Université Joseph Fourier.

${ }^{1}$ Cette disponibilité peut être considérée comme spécifique notamment parce que la réponse à l'appel d'offre n'avait pas présenté le nombre de journées engagées sur place comme le critère déterminant du prix, ce que les bureaux d'étude sont plutôt habituellement amenés à faire. Mais cette disponibilité, dans son effet participatif, peut être aussi diversement appréciée. Malgré une présence assidue aux réunions organisées sur le territoire; des échanges réguliers; des entretiens et/ou discussions téléphoniques avec toutes autres personnes souhaitant s'exprimer, des détracteurs au projet de PNR ont indiqué à l'occasion de la parution d'un article de presse (en janvier 2006), qu'ils s'étaient constitués en association (1 an après la fin de l'étude) après avoir appris l'opinion favorable au projet de PNR de personnes enquêtées lors de cette étude et « qu'ils n'avaient pas été consultés ».
} 


\section{Un diagnostic pour définir un périmètre et des actions pilotes}

Considérant la démarche de diagnostic à la fois comme préalable obligatoire à la réalisation d'un projet de territoire, celle-ci ne pouvait pas se limiter à expliciter les intentions des acteurs du territoire (phase A) et rendre compte de l'existence de ressources territoriales spécifiques et de dynamiques susceptibles d'être en adéquation avec la procédure Parc Naturel Régional (phase B). Dès le démarrage de l'étude, les commanditaires avaient donc souhaité que soit adjointe à l'issue des deux phases susdites une troisième intitulée "phase $C$ ». Celle-ci consisterait successivement: à la définition d'un périmètre de candidature à la procédure officielle, et à l'élaboration de propositions " d'actions pilotes démonstratives » répondant aux enjeux locaux et significatives quant à la capacité du territoire à s'inscrire dans un projet de type PNR. La démarche de diagnostic s'opère alors selon deux registres distincts, chacun d'entre eux étant subdivisés en étapes successives (figure 2).

Figure 2. Définition d'un périmètre de candidature et d'actions démonstratives
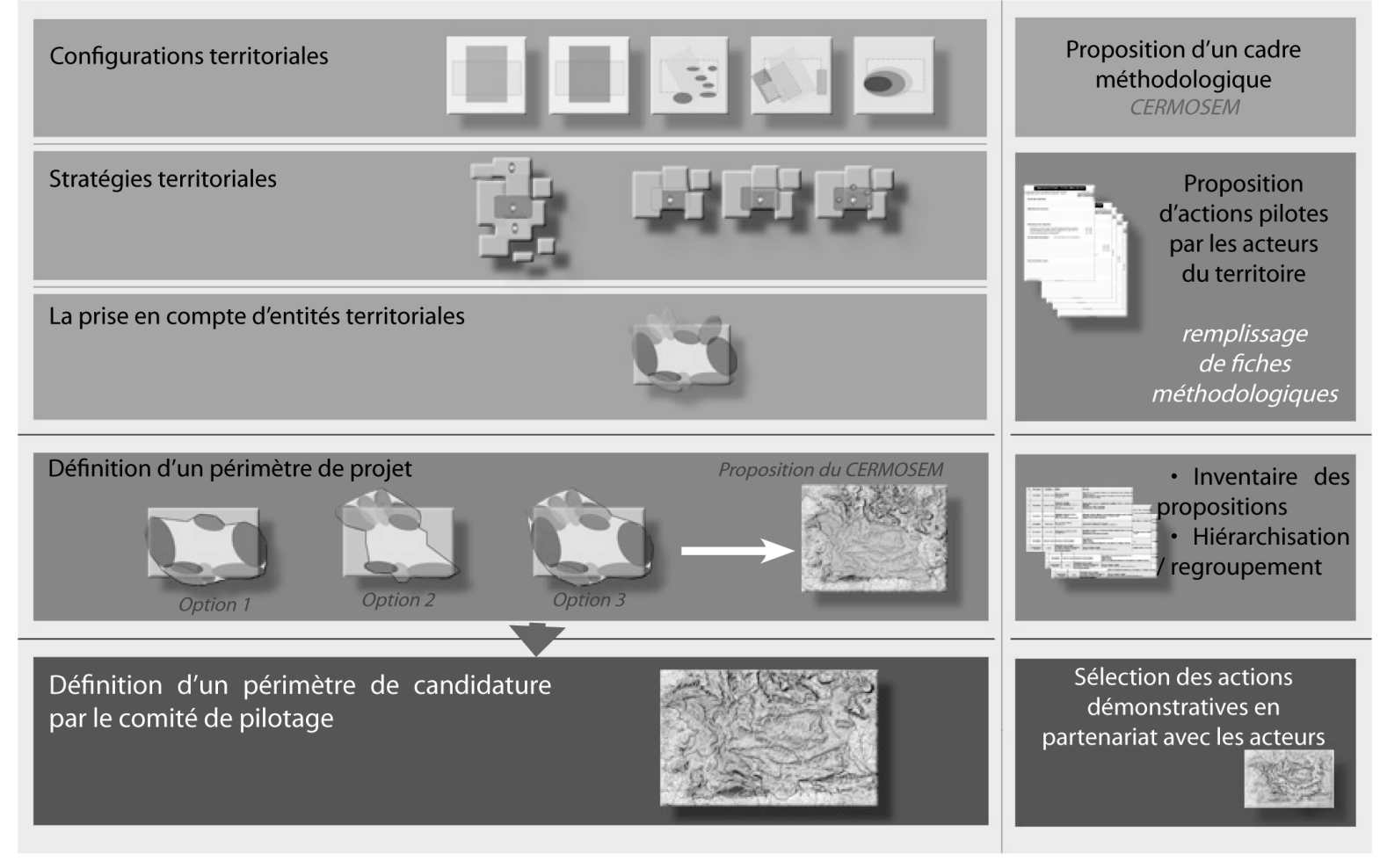

(C) E. ROUX, M. ESTERNI - I-tem Consultant, in Roux (E.), Gumuchian (H.), (dir.), 2005, p.147

\subsection{Choisir un périmètre pour le projet}

La définition d'un périmètre de projet est considérée par tous les protagonistes concernés directement ou indirectement comme l'une des phases les plus attendues et les plus sensibles également. L'objectif étant de fixer les limites à l'intérieur desquelles les communes auront à se prononcer pour ou contre le PNR, le périmètre dit " périmètre d'étude" ne pourra plus être révisé au cours de la procédure. Le périmètre doit tout à la fois être construit à partir des éléments objectifs du diagnostic (des phases précédentes) mais doit également ménager le vœu des acteurs locaux, le vœu des commanditaires soucieux de respecter une forme d'équité politique dans la représentation des deux Régions et des deux Départements, le vœu des services de l'Etat de se baser assez strictement sur des considérations objectives (et environnementales en particulier), etc. Autant dire que la question du périmètre est à la croisée d'enjeux multiples, de logiques et de stratégies d'acteurs (politiques, institutionnels) complexes et que chacun est très attentif au résultat de ce que pourra indiquer le prestataire réalisant le diagnostic. Compte tenu de ces éléments, il a été fait le choix de proposer une démarche de diagnostic reposant sur quatre types d'analyses complémentaires. 


\subsubsection{Prendre en considération les entités spatiales préexistantes}

La première analyse propose à l'aide notamment de représentations schématiques simplifiées, de rendre compte de l'existence d'entités spatiales "homogènes». Elle permettait de vérifier l'adéquation entre ces entités et quelques grandes caractéristiques de fonctionnement de l'espace des Baronnies susceptibles de guider la définition d'un périmètre d'étude. Les entités retenues concernent respectivement les cinq entrées suivantes: la "ruralité »; le fonctionnement du territoire; le patrimoine environnemental et culturel ; l'agriculture ; le sentiment d'appartenance aux Baronnies. Mais l'ensemble de ces éléments ainsi étudiés amenait à considérer que le périmètre ne pouvait certainement pas se construire à partir de la somme des critères démographiques, économiques, géomorphologiques, environnementaux, etc. S'il avait fallu retenir le plus petit dénominateur commun, le périmètre proposé n'aurait vraisemblablement satisfait personne. En revanche, ces éléments de lecture invitaient à penser que le périmètre devait tenir compte de cette mosaïque et devait exprimer un choix stratégique en privilégiant une configuration thématique plutôt qu'une autre.

\subsubsection{Analyser les enjeux de développement, de protection et de recompositions territoriales}

Dans la mesure où le choix du périmètre allait s'effectuer en fonction de choix stratégiques, il convenait de proposer une réflexion sur le sens et l'usage possible d'un Parc Naturel Régional pour le territoire des Baronnies. A partir des phases $A$ et $B$, il est rappelé sous forme de tableaux de synthèse ce que sont les enjeux associés à la protection du patrimoine naturel et culturel ainsi que ceux associés au maintien des équilibres structurants. Au terme de ces deux niveaux de priorités, il est proposé de définir un périmètre élargi proposant de prendre en compte une grande diversité patrimoniale. La définition d'un tel périmètre, de grande taille, permettait également de répondre à un grand éventail de priorités et de stratégies de développement possibles : la maîtrise foncière, l'accueil de nouvelles activités et populations, l'organisation territoriale d'un tourisme durable, la généralisation d'une agriculture de qualité et l'accompagnement territorial.

Compte tenu de l'existence d'un nombre de plus en plus grand de communautés de communes, de l'émergence de deux Pays (à l'est et à l'ouest du projet de Parc), les Baronnies provençales se trouvent dans une situation désormais classique de chevauchement ou d'entrelacement territorial. Cette configuration amène à proposer des options pour opérer la définition d'un périmètre ; deux options sont retenues, illustrées graphiquement et commentées. La première reposerait sur l'objectif de l'articulation entre les structures de coopération de niveau supracommunal (Pays, Syndicat mixte de développement) et le futur Parc Naturel Régional ; cette option imposerait une répartition claire des compétences. Dans ce cas de figure, le PNR et les Pays seraient vraisemblablement enclins à se concentrer sur leur « cœur » de territoire et cela plaiderait ainsi plutôt pour la définition d'un périmètre de Parc Naturel plutôt restreint. En revanche, la seconde option reposerait, quant à elle, sur la complémentarité entre Pays et PNR, comptant sur des projets de développement partagés et sur la nécessité de mutualisation de certains moyens d'actions. En ce concentrant sur des modalités de coopération, Parc Naturel Régional et Pays seraient vraisemblablement amenés à ouvrir des chantiers de développement communs y compris sur les marges de leurs territoires. Dans ce cas de figure, la définition d'un périmètre de Parc Naturel Régional plus étendu serait alors justifiée.

Cette définition des enjeux, adossée à une formalisation graphique, a fourni des éléments de lecture stratégique et a renvoyé les discussions sur la définition du périmètre à un débat sur la direction donnée par les acteurs locaux aux configurations territoriales souhaitées, probables et choisies.

\subsubsection{Prendre en compte l'existence d'un « cœur de Parc » et d'entités « périphériques »}

A la suite de ce travail sur les configurations et recompositions territoriales envisageables, chacun des acteurs concernés par le diagnostic de territoire s'accordait in fine à dire, qu'il existait un " cœur de Parc " et des entités (vallées adjacentes, "petit pays ") situées "en périphérie " de ce cœur. Evidemment, la discussion portait moins sur la légitimité de ce cœur que sur les périphéries possibles. Fallait-il prendre en considération la douzaine d'unités spécifiques identifiées dans la définition du périmètre de projet de Parc Naturel Régional ? Pour préparer la réponse à cette question qui s'avèrera éminemment politique, il est proposé, à l'aide d'une représentation cartographique simplifiée, de replacer ces "périphéries » dans le contexte géographique des Baronnies par rapport au cœur de PNR; puis de décliner pour chacune d'elles leurs caractéristiques et enjeux (type d'agriculture, richesse des patrimoines, etc.); et enfin de rendre compte des avantages (enrichissement patrimonial, "culture commune", lien territorial, etc.) et inconvénients (problématiques spécifiques, faible sentiment d'appartenance aux Baronnies, etc.). Ce décryptage des intérêts comparatifs permettait de les intégrer ou de ne pas les intégrer dans le périmètre du Parc Naturel Régional. 


\subsubsection{Décliner trois configurations stratégiques pour définir un périmètre et laisser émerger une quatrième option}

A partir de la déclinaison des intérêts relatifs de chacune des entités dans la définition du périmètre de Parc Naturel Régional, trois options possibles de configurations stratégiques pour définir le périmètre de projet ont été proposées. Chacune d'elle fait l'objet d'une représentation cartographique, précise les contours du périmètre retenu, explicite la prise en compte d'entités périphériques au cœur de Parc et synthétise les avantages et inconvénients associés à chaque cas de figure.

In fine, après présentations de ces options, les commanditaires et acteurs du territoire, à force de débats, de négociations sur le fond comme sur la forme, d'arbitrages, ... ont opté pour une quatrième option. Celle-ci s'appuie, certes, sur les éléments d'analyses proposés, mais correspond plus résolument à l'accord obtenu entre les parties en présence dans le jeu, à savoir les élus du territoire et les commanditaires. Notre propos privilégiant une dimension méthodologique, ce qui semble intéressant dans cette expérience relève de la démarche et du procédé de construction du diagnostic permettant aux acteurs locaux de ne pas être (trop) dessaisis de la description pour leur permettre d'être complètement saisi par les enjeux de la définition stratégique du périmètre. A ce moment-là, le système d'acteurs locaux se trouve en avance d'une négociation puisqu'ils sont d'accords sur l'intérêt de la démarche Parc, d'accords sur ce qui constitue le « cœur de PNR » (autant du point de vue de la position dans l'espace que de son identification à des problématiques de développement) et ils préparent l'accord sur ce qui va constituer leurs alliances principales avec les territoires voisins dans le futur.

\subsection{Un dispositif pour choisir des actions pilotes et ainsi favoriser l'émergence d'intentions d'actions}

La détermination d'un périmètre de candidature était certes une étape incontournable et cruciale de tout le processus qui s'est proposé de mettre en place une démarche itérative plutôt que de se focaliser sur la justification d'un périmètre idéal ; mais une autre facette de la démarche de diagnostic était tout aussi attendue : celle de la mise en œuvre d'actions démonstratives. Là aussi, il a été préconisé de mettre en place une démarche en trois étapes qui favorise l'expression par les acteurs du territoire de leurs intentions d'actions.

\subsubsection{Rappeler le sens des actions pilotes démonstratives}

La première consistait à rappeler aux acteurs du territoire les règles du jeu et le sens des actions que pouvait porter un Parc Naturel Régional. II s'agissait d'une part de rendre compte au travers d'exemples pris dans d'autres PNR, de ce que peuvent être ces actions et d'autre part d'énoncer quelques principes à considérer pour la définition et la mise en œuvre de ces dernières (transversalité, démonstrabilité, diversité, représentativité territoriale, ...).

\subsubsection{Faciliter l'émergence de propositions issues du territoire}

La seconde proposait un dispositif facilitant l'émergence de propositions d'actions par les acteurs du territoire : une fiche méthodologique permettant la description de la ou des actions (objectifs, résultats attendus, publics visés, porteurs du projet, partenaires...) a alors été élaborée. Celle-ci a ensuite été diffusée auprès des acteurs du territoire (collectivités territoriales, structures de développement, chambres consulaires, associations, fédérations, ...). Dès lors, ces derniers ont été invités à présenter leurs projets.

\subsubsection{Pour permettre la sélection et le choix d'actions pilotes}

La troisième étape correspondait à la sélection et le choix des opérations. II s'agissait, à partir des propositions reçues, de procéder à des regroupements, de vérifier leur adéquation aux principes énoncés en amont, de proposer une sélection d'opérations et de soumettre celle-ci au Comité de pilotage.

Le choix des actions proposées et retenues a été opéré par les acteurs du territoire et les commanditaires du diagnostic. Ainsi, si le prestataire réalisant le diagnostic a bien effectué une proposition qui s'appuie sur les éléments issus du territoire, le choix final demeure là également le fruit d'une négociation entre les régions et les acteurs locaux : les Régions ont retenu telles actions plutôt que d'autres (notamment en mettant l'accent sur la préservation des patrimoines naturels) et les 
acteurs du territoire ont souhaité retenir des actions permettant du développement, du maintien de l'agriculture, de la maîtrise du foncier, etc. Les protagonistes du projet (commanditaires et acteurs locaux) ont échangé, ont travaillé ensemble et le prestataire pouvait alors prendre acte des choix, éventuellement les formaliser, puis conclure l'étude.

\section{Proposition pour une analyse de la démarche de diagnostic dans la construction d'un projet de territoire}

Analyser la signification du diagnostic et de la manière dont se fabriquent progressivement, en une petite année, au cours de cet exercice quelques unes des conditions de recevabilité d'un projet de PNR est notre intention première. Mais l'observation de cette participation pourrait être enrichie par l'analyse par exemple des " plus-values de cette posture de recherche-participante " ou encore la relecture des influences multiples de cette pratique sur les acteurs locaux et les commanditaires. Notre propos s'est ici concentré sur la pratique du diagnostic et son usage dans le processus de construction territoriale. Et à partir de cette expérience, il est proposé une grille d'analyse offrant le moyen de la comparaison de cas de figure et considérant l'intérêt des diagnostics de territoires en les jaugeant à l'aune de leurs processus « instituant » et « constituant».

\subsection{Paradoxes du diagnostic et processus de territorialisation par le diagnostic}

De cette pratique effective de diagnostic de territoire orienté vers l'action à court terme, nous pouvons tirer quelques remarques terminologiques et quelques propositions analytiques pour tenter d'éclaircir le paradoxe apparent du diagnostic. Le recours, somme toute assez classique, à la métaphore médicale permet d'apporter quelques éclairages quant au sens de la démarche de diagnostic. A priori, celle-ci relève de « l'exceptionnalité ». II est certes possible de consulter son médecin régulièrement mais on attend de ce dernier " un diagnostic » pour expliquer un état exceptionnel ou pour contrôler un état particulier. Le médecin traitant fonde la légitimité de son diagnostic sur une connaissance plus ancienne, plus globale et en même temps plus profonde de son patient, ou d'un patient " qui lui ressemble ». De façon logique, le diagnostic invite implicitement à considérer qu'il existe potentiellement un dysfonctionnement ; tout en espérant vivement que " tout aille bien ». En tout état de cause, s'opère par ce dernier une forme de comparaison entre l'état particulier constaté, " horsnorme » et un état que l'on considèrerait comme "plus normal ». En quoi le diagnostic de territoire relève-t-il également de ce principe? Le territoire diagnostiqué est-il pensé explicitement ou implicitement par rapport à un territoire de référence donné, en bon état, " plus normal » ? Ici, la référence est contenue dans le "modèle » territorial "Parc Naturel Régional » largement validé et confirmé par les quarante ans d'existence institutionnelle de ce type de territoire. Dans ce contexte, si l'objectif est bien d'aller vers la mise en place d'un Parc Naturel Régional, le diagnostic, envisagé comme un élément premier de ce long processus, ne pourrait que difficilement aboutir à constater que les Baronnies ne ressembleraient pas exactement à un territoire susceptible de devenir Parc Naturel Régional un jour. Mais, il est admis que chaque territoire-Parc doit développer sa spécificité ; autrement dit que ce qui ferait la spécificité (ou la particularité hors norme) de ce territoire constituerait effectivement un des arguments principaux de son inscription dans une procédure de type Parc Naturel Régional. II existe donc là une ambiguïté certaine consistant à faire dire à la fois au diagnostic que ce territoire est comparable à celui d'un Parc sans jamais être réduit seulement à cela. La démarche de diagnostic s'avère ainsi tout à la fois stratégique et/car périlleuse. Si le travail de diagnostic consiste à dire avec le plus d'exactitude et de vraisemblance possible ce qu'est ce territoire, la probabilité de venir en contradiction avec le « modèle Parc » risque d'être grande et la procédure de classement ministériel risque par conséquent d'être vouée à l'échec. Par contre, si le diagnostic aboutit à constater que tous les critères attendus pour « faire Parc » sont ici réunis, la probabilité d'être en contradiction avec ce que les acteurs locaux veulent vraiment risque également d'être grande et la procédure de classement est alors également susceptible d'être vouée localement à l'échec.

Ce paradoxe apparent pourrait être résolu en proposant que le processus dans lequel se trouve inséré le diagnostic procède de manière évidente de celui de la reterritorialisation. Or dans les Baronnies, depuis une vingtaine d'années, de multiples territorialisations (projets de développement, structures et organisations assurant du développement, nombreuses procédures obtenues pour financer du développement...) sont à l'œuvre. Elles portent résolument la conception du développement qui est celle prônée dans les PNR. Tout l'enjeu ici est de comprendre comment ce processus de 
territorialisation par le diagnostic se déroule. Celui-ci s'opère selon nous selon trois étapes complémentaires (cf. figure 3) :

- la première consiste en un travail sur une appréhension « nouvelle » des choses et des acteurs ainsi que sur un remodelage des représentations collectives, une re-connaissance,

- la seconde correspond à la construction d'une perspective pragmatique (la construction d'un Parc Naturel Régional) assise sur une opérationnalité potentielle,

- la troisième aboutissant à une reconfiguration des jeux d'acteurs permettant de faire émerger de nouvelles manières d'instituer des référents innovants et de constituer un territoire nouveau.

\section{Figure 3. Du diagnostic pour territorialiser}

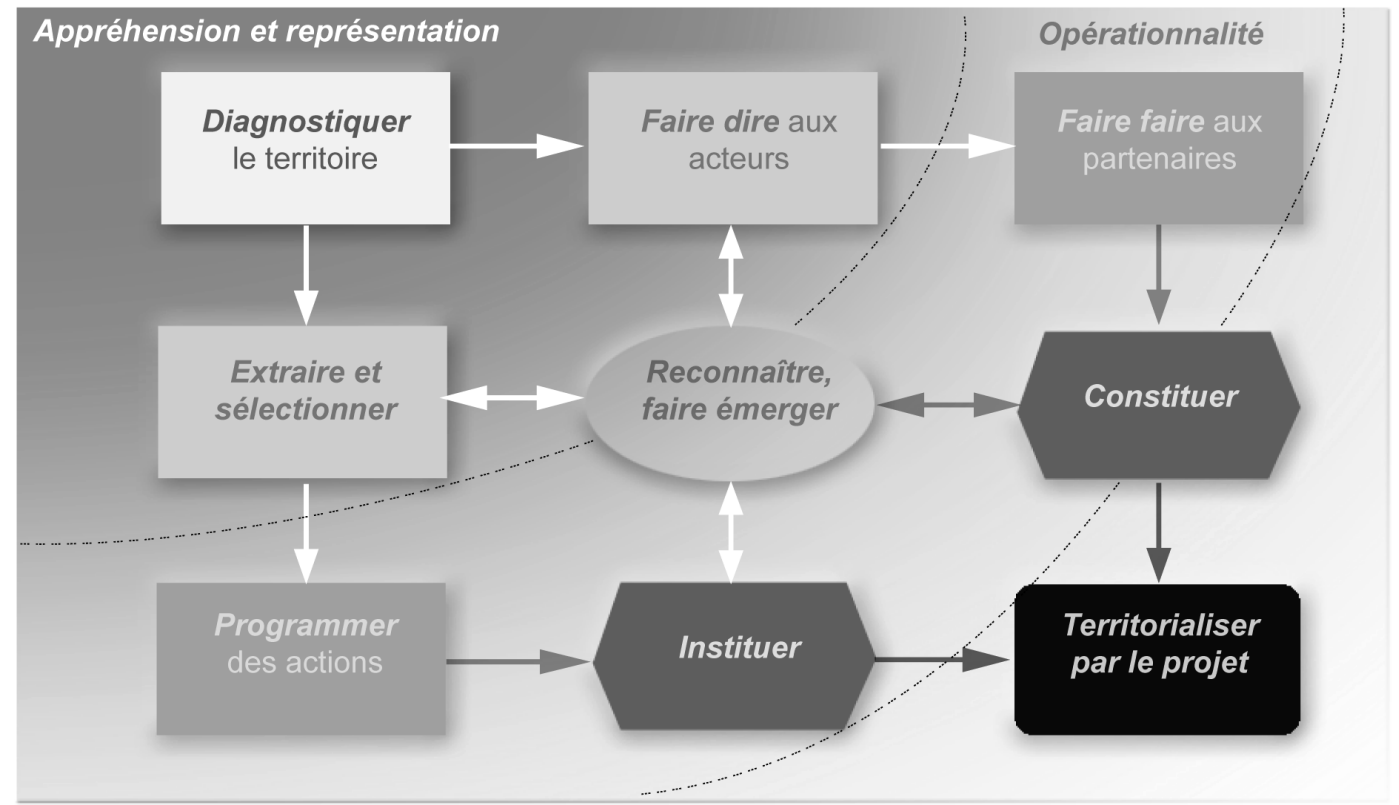

(๑) R. LAJARGE, M. ESTERNI - I-tem Consultant, 2005

\subsection{Diagnostiquer pour appréhender et représenter autrement}

La phase de diagnostic a consisté, de la part du prestataire, à extraire de l'ensemble des données qu'il a pu mobiliser ou rassembler, celles qui semblent les plus parlantes pour le commanditaire (et de préférence parlantes également pour les acteurs du territoire) et celles qui ont la plus grande valeur explicative du fonctionnement du territoire. La phase de diagnostic consiste donc à s'appuyer sur ce qui rend spécifique le territoire, sur ce qui le différencie dans son essence de tout autre territoire, bref sur une vision particulariste du réel qui permet aux acteurs territorialisés de le re-connaître. Mais une fois le travail de spécification terminé, le territoire doit se faire reconnaître par les institutions ; il lui faut donc ressembler au modèle de référence et aux principes qui sont associés à ce modèle de référence. La phase de construction territoriale consiste donc à démontrer ce qu'il y a de générique dans ce territoire, ce qui le rend similaire (assimilable par les semblables) à d'autres. Bref, le territoire doit également s'appuyer sur une vision partageable du réel qui permet aux acteurs régionaux et nationaux de le reconnaître en le labellisant. Cette mise en tension entre vision particulariste et vision générique dit bien le paradoxe dans lequel se trouve l'exercice du diagnostic de territoire.

Dans cet exemple du territoire des Baronnies, la description de la déprise rurale que l'on peut constater permet de conclure à la fragilité d'un tel territoire ; la description de la fragilité est notamment traduite par des indices forts de vieillissement de la population, des faibles densités de population, de faibles taux d'activités non agricoles, etc. Mais cette fragilité se traduit dans des faits, dans des récits et des interprétations, dans des trajectoires d'individus comme de familles qui sont particulières aux baronnies provençales. En même temps, ce territoire connaît une forte attractivité (augmentation significative du prix du foncier, arrivée de nouvelles populations, etc.) qui se traduit dans l'expression langagière " de luberonnisation ", peu synonyme de pauvreté tant le Luberon est plutôt reconnu pour 
accueillir une population résidentielle et touristique à hauts revenus, comme c'est le cas, de manière générique dans de nombreux endroits de la Provence soumis à l'héliotropisme.

Ce travail d'extraction et de sélection aboutit quasi-inéluctablement au final à la proposition par les acteurs du territoire d'une multiplicité de propositions très concrètes d'opérations à mettre en œuvre pour démontrer leur capacité à devenir Parc Naturel Régional. Mais même si le réalisateur du diagnostic tente d'opérer consciencieusement une analyse du territoire, d'argumenter cette dernière, s'efforcer de mettre en évidence ses conclusions au travers de grilles de lecture, de graphiques, de représentations cartographiques; force est de constater qu'une production ex-nihilo n'a, dans un temps court, que peu d'effets sur l'appréhension du réel et le remodelage des représentations locales. Cet état de fait invite dès lors à considérer que la réalisation du diagnostic a d'autant plus d'effets qu'elle exprime le souci de la prise en compte des acteurs du territoire. Comme le propose Sylvie Lardon à propos des méthodes graphiques dans la construction des diagnostics, il s'agit donc de " fournir aux acteurs les outils de leur propre raisonnement " ${ }^{1}$. Dans ce contexte, " faire dire " relève selon nous, tout autant d'un principe méthodologique que d'un principe éthique. Les destinataires du diagnostic, faut-il le rappeler, ne sont pas seulement les commanditaires; un tel travail doit tout à la fois "servir " dans la procédure et "servir " localement. Le diagnostic est en effet destiné à déclencher de nouvelles manières de décrire le réel, de la part d'acteurs directement concernés par la procédure, sans qu'ils aient eu besoin de posséder effectivement l'ensemble du document du diagnostic (trop volumineux pour faire l'objet d'une large diffusion, trop codé pour faire l'objet d'une édition). Une fois que les conclusions du diagnostic sont diffusées (ou même parfois que très partiellement diffusées), les acteurs locaux et régionaux vont pouvoir enclencher la démarche de " faire faire ». II s'agit alors, comme dans la procédure des Baronnies avec les opérations pilotes, de faire réaliser par d'autres (chambres consulaires, associations, collectivités, structures de développement, porteurs de projets...) des actions précises en s'appuyant sur la justification que les leaders du projet ont énoncé entre eux.

Parce que les protagonistes du projet de Parc constatent que les Baronnies sont fortement dépendantes de la maîtrise dans l'avenir de la spéculation foncière, ils vont inciter des opérateurs directs de cette question à penser « ce qui serait souhaitable de faire " pour trouver une solution à ce problème. Elus locaux, instructeurs de procédures d'urbanisme, établissement foncier régional, structures de développement, etc. ; tous sont ainsi invités à se joindre à cette nouvelle représentation territoriale de ce que sont les Baronnies. Dans notre cas de figure, en faisant le constat de reprise résidentielle non maîtrisée face à une inévitable déprise rurale traditionnelle, le processus de diagnostic va rendre difficilement recevables les raisonnements (classiques dans les espaces ruraux en crise) indiquant qu'il existe exclusivement un abandon, une désertification ou un déni.

\subsection{Se faire reconnaître en diagnostiquant pour agir et faire agir}

Dans une acception plus strictement technicienne et/ou peut-être de façon plus naïve ${ }^{2}$, « dire ce qui est ", même en amenant des preuves les plus tangibles possibles, ne permet guère de faire émerger d'autres formes de représentations collectives vraiment opérantes. Au mieux, en montrant une autre figuration de l'existant, on attise l'envie de faire dire par d'autres catégories d'acteurs ce qu'il faudrait changer. Pour poursuivre notre exemple, en montrant que derrière l'apparence de la déprise, une reprise est en cours, on attise l'envie de travailler sur des modalités d'accueil de nouveaux habitants et on minimise les propos sur la perte de la terre, sur la défense de la propriété privée ou de la chasse.

Le diagnostic territorial est donc souvent un instigateur de changement social local. En le conduisant sur le terrain avec des acteurs préalablement repérés, comme prêts à agir et à piloter le changement, (de façon co-construite), on augmente non seulement l'efficacité du diagnostic mais aussi sa pertinence. Et si le constat est fait d'absence d'acteurs mobilisables, il est certainement préférable de s'abstenir de « le dire », ce qui est probablement éthiquement contestable, mais il serait de toute façon irresponsable politiquement de « tout dire ». Dans les Baronnies, par exemple, l'évidence du problème naturaliste rend périlleux d'aborder la spécificité du territoire par la richesse reconnue de son patrimoine naturel protégé. Le danger vient moins de l'opposition frontale et radicale exprimée à la seule évocation de dispositifs type Natura 2000 ou des Réserves Naturelles que de la confrontation des représentations sur ce qui fait effectivement la richesse patrimoniale de ce territoire. Pour se faire reconnaître comme opérant, le diagnostic doit donc plutôt évoquer la «'mosaïque d'habitats', [...] la

\footnotetext{
${ }^{1}$ Lardon (S.), 2003, p127

${ }^{2}$ Cet argument est retrouvé parfois dans certains exercices de diagnostics, de temps en temps dans des rapports de stage d'étudiants et le plus souvent dans des travaux scientifiques de type positivistes qui croient encore que la description fine d'objets spatiaux suffit à dire ce que sont ces objets, nonobstant l'usage qu'en font les sociétés en se les appropriant notamment par les représentations.
} 
richesse des nombreuses espèces 'patrimoniales', [...] l'importante diversité locale, ... » qui permettent de dire in fine que "L'identité des Baronnies réside dans cette singularité. Ce secteur est une unité écologique en soi, caractérisée par une complexité locale importante ${ }^{1}$. Bien entendu, les expressions "mosaïques ", " espèces patrimoniales " ou encore "zones ouvertes " ont l'indicible avantage d'être acceptables à la fois par les écologistes et les chasseurs, d'être des références à la fois à la biodiversité environnementaliste et aux préconisations cynégétiques, d'être connectées aux souhaits de protéger plus encore le patrimoine des uns et aux autres de leur garantir de continuer à accéder de manière privilégiée à ce patrimoine. L'adhésion des uns et des autres au projet de Parc Naturel Régional est suspendue à cette figure particulière de rhétorique qui permet à la fois de dire les choses, de partager un point de vue sur ces choses et d'envisager de programmer les actions que ces choses dites permettent.

\section{Conclusion : territorialiser par le projet en instituant et en constituant}

Pour que la territorialisation soit effective, il ne s'agit pas seulement de se satisfaire de la dimension performative du diagnostic territorial. A un moment du processus, le programme d'action qui se dessine (l'agir) et le programme de coopération qui est son pendant (le faire faire), ne suffisent pas. Les acteurs locaux comprennent et admettent (plus ou moins) que le diagnostic se concrétise matériellement, puisque telle est la règle, par la production d'un document comportant du texte, des grilles de lecture, des analyses de données, des analyses de discours, d'actions programmatiques, des représentations cartographiques, etc. Mais cette seule perspective ne saurait au bout du compte satisfaire chacun. Les acteurs locaux souhaitent aussi et surtout que le diagnostic trouve un aboutissement dans la réalisation concrète "d'actions démonstratives " sur le territoire. Ainsi, dans les Baronnies, les acteurs locaux réclament aux acteurs régionaux les preuves manifestes de l'inscription de cette démarche de réflexion dans une démarche de réalisation. Dans notre cas de figure, la troisième phase de cette étude avait exactement pour objet d'assurer cette transition : se doter des moyens de réaliser l'intention, tel est le pari de la sortie du diagnostic. Cette perspective (nouvelle) associée à la démarche de diagnostic peut se réaliser de deux manières.

Instituer. La perspective d'aboutir un jour ou l'autre à des actions propres au territoire, nouvelles et par postulat innovantes, oblige les acteurs locaux à proposer de mettre en place quelque chose de nouveau (un dispositif) qui aurait un début attesté. Cet addendum nécessaire, dont il faudrait concevoir les modalités spécifiques, les responsabilités politiques, les moyens techniques a pour vocation que chacun puisse constater l'avènement du projet. Les « opérations pilotes » ont cette vertu d'instituer un nouveau rapport à l'action, avec un nouveau référentiel et de nouvelles visées. Cette démarche d'institutionnalisation est la marque de ce qui relève de l'action et de ce qui est sujet au changement, à l'adéquation permanente au projet. Instituer les enseignements tirés du diagnostic consiste donc à alimenter la démarche de projet par des dispositions pratiques. Effectivement, la perspective de montrer concrètement sur une quarantaine de sujets, ce que pourrait être le contenu de la future charte relève bien de cette envie de voir progresser ensemble les représentations du territoire et les actions sur ce territoire.

Constituer. La territorialisation par le projet ne peut se résumer en une mise en application partielle et préalable de quelques unes des orientations souhaitables démontrées par le diagnostic et instituées dans un nouveau dispositif d'action collective organisée. La territorialisation implique une reconnaissance politique formelle et une traduction dans des instances nouvelles de la représentation politique de ce que le territoire construit. Ce travail revient à constituer autour du projet un accord global et légal.

Cette " constitution » est nécessaire car il s'agit d'inscrire le constat de la territorialisation naissante dans une stabilité, de façon fixe et sûre. Et ce processus passe par exemple dans les Baronnies par des débats toujours longs sur la nécessaire création d'un syndicat mixte, avec son bureau, ses statuts, avec les demandes d'adhésion et le financement public qui va avec. Un accord global est également nécessaire car la constitution des territoires engage non seulement l'intérêt général mais tout un chacun. Cet accord global doit alors être double : il est légitime et indispensable du point de vue démocratique, mais il est également important du point de vue citoyen si l'on souhaite que le projet soit largement partagé. L'accord doit être légal car la nature du processus de territorialisation va

\footnotetext{
${ }^{1}$ Cf. paragraphe 2.4. page 106 du rapport d'étude (partie 2, Faisabilité) in E. Roux, H. Gumuchian, ss. Dir. «La construction d'un projet de territoire. Un projet de Parc Naturel Régional pour le territoire des Baronnies », UMR Pacte, CermosemTerritoires, 2005.
} 
engager des parties et des institutions qui auront nécessairement des intérêts et des positions différentes dans le projet. Les éventuels désaccords, litiges ou mésententes seront donc susceptibles d'être renvoyées aux formulations explicites du contrat qui accompagnera le projet.

In fine, la démarche de diagnostic est susceptible d'aboutir dans le cas des Baronnies à un contrat ; et s'il y a " contrat ", il devient obligatoire de constituer fixement les rôles de chacun, bref d'inscrire dans la stabilité d'une constitution l'accord qu'exprimera le projet.

Pour que le diagnostic de territoire passe du statut d'artefact à celui d'outil politique, il lui faut s'arrimer au processus de la reterritorialisation non seulement dans ces trois dimensions symboliques, cognitives et rhétoriques mais également dans sa dimension pratique, programmatique et organisée. C'est parce le diagnostic de territoire ne permet pas à lui seul de «reconnaître » et de "faire émerger » des représentations nouvelles permettant de réorganiser l'action locale, qu'il lui faut à la fois traduire, sélectionner, hiérarchiser et en même temps faire dire de multiples manières par d'autres ce qui est à l'œuvre dans ce territoire. Même si le diagnostic parvient à montrer, de manière apparemment objective, ce qui serait judicieux de choisir comme stratégie dans tel ou tel type de territoire, et même s'il parvenait à faire émerger un groupe d'acteurs reconnus comme porteurs de cette stratégie, il ne serait encore pas possible d'en déduire directement les formes, structures et principes du territoire de projet qui en sortirait. Sans l'institution de modalités nouvelles de pilotage du changement et sans la constitution d'un accord contractualisé entre des acteurs renouvelés, le diagnostic de territoire ne produit que les artefacts de la territorialisation, les prémisses d'une mise en mouvement autour du projet, les étincelles cognitives dans la fabrique des territoires (Vanier M., 1995 ; Antheaume B., Giraut F., Ed., 2005). Par contre, si à l'occasion du diagnostic, les acteurs récupèrent les bribes utiles d'une argumentation nouvelle, les indicateurs objectifs d'enjeux renouvelés ou les occasions de coopérations-partenariats d'un nouveau genre, alors le diagnostic de territoire est partie prenante du processus de territorialisation et devient un outil politique ; alors la perspective de le voir s'empoussiérer sur l'étagère d'un commanditaire pressé est remplacée par celle, peut-être plus réjouissante pour la recherche impliquée, d'une pièce à conviction supplémentaire prouvant la patiente et continuelle gestion que les acteurs font de la complexité territoriale.

\section{Eléments de bibliographie}

Antheaume (B.), Giraut (Fr.) (eds), 2005, « Le territoire est mort. Vive les territoires! Une (re)fabrication au nom du développement $"$, éditions IRD

Boutinet (J.-P.), 1993, "Anthropologie du projet», Paris : éd. PUF, coll. Psychologie d'aujourd'hui (1ère édition 1990), 312p.

Comité de Liaison des Comités de Bassins d'Emploi, DATAR, 1997, «Construire un projet de territoire. Du diagnostic aux stratégies ", éd. Ministère de l'Equipement-DAFU, éditions Villes et territoires, sous la coordination de Mazel (O.), Vazard (P.), Wener (K.), 53p.

Conseil Général du Génie Rural, des Eaux et des Forêts, 1993, "Guide méthodologique du développement territorial », Paris : éd. Ministère de l'agriculture et de la pêche, $51 \mathrm{p}$

Corboz (A.), 2001, "Le territoire comme palimpseste », éd. Les éditions de l'imprimeur, collection tranches de villes, recueil de textes présenté par Sébastien Marot, 281p.

Cuaresma (M.), Pecqueur (B.), 1997, « Mener un projet de développement local », Paris : éd. Les éditions d'organisation, $115 \mathrm{p}$.

Debarbieux (B.), Lardon (S.) (dir.), 2003, « Les figures du projet territorial », éd. de l'Aube-DATAR, $269 p$.

Giraut (F.), Vanier (M.), 1999, « Plaidoyer pour la complexité territoriale », in Gerbaux (F.) (2000) (dir.), "L'utopie des territoires: cohérence ou complexité ? », éd. de l'Aube, coll. Territoires, pp. 143-172

Guéringer (A.), Perret (J.), Baud (P.), 1993, « Diagnostics à l'échelle de petites régions rurales: démarches, méthodes et sources d'informations », Coll. Etudes du CEMAGREF, Série Production et Economie agricoles, $\mathrm{n}^{\circ} 5,196 \mathrm{p}$.

Gumuchian (H.), Grasset (E.), Lajarge (R.), Roux (E.), 2003, « Les acteurs, ces oubliés du territoire », Coll. Géographie, Anthropos, Paris, Ed. Economica, 186 p.

Lajarge (R.), 2000, "Territorialités intentionnelles. Des projets à la création des Parcs naturels régionaux (Chartreuse et Monts d'Ardèche) ». Thèse de doctorat, Géographie, Université Joseph Fourier, $663 \mathrm{p}$.

Lajarge (R.), Roux (E.), 2004, « L'activation des ressources par le projet : les Baronnies en recherche de nouvelles légitimités », in "La notion de ressource territoriale », Montagnes Méditerranéennes, $\mathrm{n}^{\circ} 20$, pp. $165-176$ 
Lardon (S.), 2003, "Diagnostic de territoire et représentations spatiales », in Debarbieux (B.), Lardon (S.) (dir.), 2003, « Les figures du projet territorial », éd. de l'Aube-DATAR, pp109-129

Mairie-Conseil, 1997, "La Charte de territoire. Une démarche pour un projet de développement durable. ", Paris : éd. La Documentation Française, 311p.

Rigaldies (B.), 1996, « Le projet de territoire », Montreuil : éd. du Papyrus, coll. Guide Actelus, 117p.

Roux (E.), Gumuchian (H.), (dir.), 2005, «La construction d'un projet de territoire. Un projet de Parc Naturel Régional pour le territoire des Baronnies », CERMOSEM, UMR Pacte, Territoires, $230 p$

Vanier (M.), 1995, "La petite fabrique de territoires en Rhône-Alpes : acteurs, mythes et pratiques ", in Revue de Géographie de Lyon : Nouvelles mailles du pouvoir local, $n^{\circ}$ spécial, volume $70, n^{\circ} 2$, pp. 93-104 